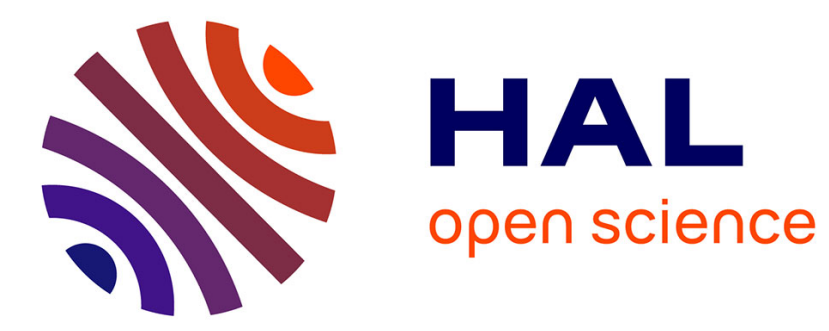

\title{
Le bilan du moment cinétique et les règles de polarisation en spectroscopie. Application à la diffusion de la lumière et à la fluorescence
}

A. Kastler

\section{- To cite this version:}

A. Kastler. Le bilan du moment cinétique et les règles de polarisation en spectroscopie. Application à la diffusion de la lumière et à la fluorescence. J. Phys. Radium, 1933, 4 (7), pp.406-420. 10.1051/jphysrad:0193300407040600 . jpa-00233161

\section{HAL Id: jpa-00233161 https://hal.science/jpa-00233161}

Submitted on 1 Jan 1933

HAL is a multi-disciplinary open access archive for the deposit and dissemination of scientific research documents, whether they are published or not. The documents may come from teaching and research institutions in France or abroad, or from public or private research centers.
L'archive ouverte pluridisciplinaire HAL, est destinée au dépôt et à la diffusion de documents scientifiques de niveau recherche, publiés ou non, émanant des établissements d'enseignement et de recherche français ou étrangers, des laboratoires publics ou privés. 


\title{
LE BILAN DU MOMENT GINÉTIQUE ET LES RĖGLES DE POLARISATION EN SPECTROSCOPIE (*)
}

\section{APPLIGATION A LA DIfFusion DE LA LUMIĖRE ET A LA FLUORESCENCE}

\author{
Par A. KASTLER,
}

Assistant à la Faculté des Sciences de Bordeaux.

\begin{abstract}
Sommaire. - Après avoir indiqué comment le principe de conservation du moment cinétique et les règles de polarisation s'appliquent en spectroscopie en absence de champ extérieur, l'auteur donne des exemples d'application :

10 A la diffusion de la lumière. - Dans ce cas, les résultats concordent avec ceux de la théorie classique.

$2^{\circ}$ Aux phénomènes de fluorescence obtenus par excitation optique des atomes par échelons.
\end{abstract}

Le principe de conservation du moment cinétique a été introduit en spectroscopie par Rubinowicz $\left.{ }^{1}\right)$ qui en a déduit les règles de sélection et de polarisation qui régissent les échanges d'énergie entre l'atome et la radiation $\left(^{2}\right)$. Le moment cinétique d'une orbite électronique dans un niveau quantique est :

$$
\vec{M}=l \cdot \frac{h}{2 \pi}(l=\text { nombre quantique azimutal }) .
$$

La lumière possède un moment einétique nul lorsqu'elle est polarisée rectilignement. Lorsqu'elle est polarisée circulairement, à droite ou à gauche, son moment cinétique est égal à :

$$
\vec{\mu}= \pm \frac{h}{2 \pi}
$$

(les signes + et - désignant des moments cinétiques respectivement dextrorsum et sinistrorsum).

La conservation du moment cinétique entraîne donc :

$1^{\circ}$ La règle da sélection : $\Delta l=0$ ou $\Delta l= \pm 1$.

$2^{\circ}$ La règle de polarisation : dans l'émission la transition $\Delta l=0$ fournit de la lumière rectiligne, les transitions $\Delta l= \pm 1$ fournissent des lumières circulaires, respectivement droite ou gauche, (dans l'absorption elles correspondent à des lumières circulaires gauche ou droite).

La règle de sélection a été étendue à la variation du nombre quantique interne $j$ : $\Delta j= \pm 1$ ou 0 et à celle du quantum équatorial ou magnétique $m: \Delta m= \pm 1$ ou 0 .

La règle de polarisation a trouvé des applications dans les effets Zeeman et Stark. Lorsque l'atome est soumis à un champ extérieur, magnétique ou électrique, la quantification spatiale a lieu et la composante $\vec{m} \cdot \frac{h}{2 \pi}$ du moment cinétique de l'atome suivant le champ est seule définie et constante. Dans ce eas, c'est au seul nombre quantique $m$ (quantum

( $^{\star}$ Mémoire exposé à la Société de Sciences Physiques et Naturelles de Bordeaux, séance du 18 mai 1933. 
équatorial ou magnétique) que s'appliquent les règles de polarisation. Dans l'effet Zeeman, les transitions $\Delta m=0$ et $\Delta m= \pm 1$ sont séparées par un appareil dispersif et présentent bien chacune l'état de polarisation exigé.

La figure 1 donne le schéma Zeeman de la raie de résonance du mercure $\left({ }^{2} \boldsymbol{P}_{1}-1^{1} S_{0}\right)$, transition entre les niveaux $1^{1} S_{0}(j=m=0)$ et $2^{3} P_{1}(j=1 ; m=-1,0,+1)$. Dans ce schéma les niveaux sont représentés par des lignes horizontales sur lesquelles sont marqués les nombres $m$. Les transitions d'un niveau à l'autre sont représentées par des traits : un trait vertical correspond à de la lumière rectiligne, un trait oblique à de la lumière circulaire droite ou gauche, suivant que sa pente est positive ou négative.

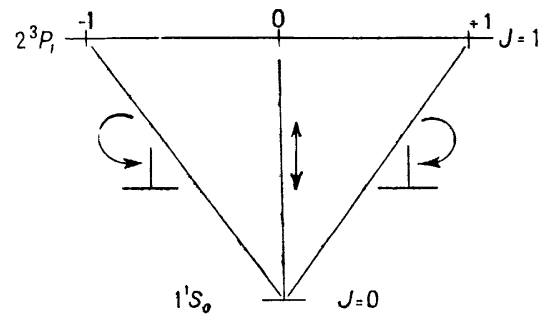

Fig. 1.

Lorsqu'il n'y a pas de champ extérieur, les règles de sélection s'appliquent toujours, mais il semble à première vue, qu'on ne puisse rien tirer de la règle de polarisation; ainsi Sommerfeld écrit en 1922 (Atombau u. Spektrallinien, $3^{\mathrm{e}}$ édition, p. 331):

« Offenbar entzieht sich beim kräftefreien Atom die Polarisationsregel dem Experimente. Hier ist jede Lage im Raum mit jeder anderen gleichberechtigt. Wenn also auch der Einzelvorgang je nach der Lage des Atoms polarisiert ist, so wird doch die beobachtbare Gesamterscheinung vollkommen depolarisiert sein ).

En efiet, en l'absence de champ extérieur, la quantification spatiale disparaìt et les atomes sont orientés au hasard. Il est apparu cependant, que la conclusion précédente était trop pessimiste. Dans l'émission de lumière, la cause d'émission étant en général isotrope, la règle de polarisation ne peut pas s'appliquer, mais il n'en est pas de même dans les phénomènes de fluorescence ou de diffusion de la lumière où les atomes sont soumis à une onde incidente de direction déterminée. Dans ce cas, les éléments de symétrie de la lumière incidente constituent, pour le phénomène, des directions privilégiées dans l'espace, et se retrouvent dans l'onde réémise ou diffusée.

Le moment cinétique d'un atome est caractérisé aujourd'hui, par les nombres quantiques $L$ (quantum azimutal), $J$ (quantum total), et $M$ (quantum magnétique). Ces nombres, considérés comme des vecteurs dans l'espace, $\vec{L}, \vec{J}$ et $\overrightarrow{\|}$ représentent en grandeur et en direction, le moment cinétique correspondant en unités $\frac{h}{2 \pi}\left({ }^{\star}\right)$. Comme il n'y a pas de quantification spatiale en l'absence de champ directeur, la question qui se pose est de savoir auquel de ces trois vecteurs il faut appliquer alors le bilan de moment cinétique et les règles de polarisation. Cette question a été résolue par Heisenberg $\left({ }^{3}\right)$, qui a montré la validité du postulat suivant : tout se passe en l'absence de champ extérieur, comme si la quantification spatiale des atomes existait par rapport à un champ fictif. Si la lumière incidente est polarisée rectilignement, ce champ fictif est confondu avec le vecteur électrique des vibrations

(*) Il est fait ici abstraction d'une complication introduite par la mécanique ondulatoire selon laquelle Je moment cinétique serait $\sqrt{J(J+1)}$ au lieu de $J$. 
incidentes. Si la lumière incidente est polarisée circulairement, il est dirigé suivant la normale à l'onde. Dans les deux cas, cette direction de quantification apparente est donc un axe de révolution de la vibration incidente. Il en résulte qu'en absence de champ extérieur c'est encore au quantum magnétique $\mathrm{M}$ - et non pas au quantum total $\mathrm{J}$, comme on pourrait s'y attendre au premier abord - qu'il faut appliquer le bilan de moment cinétique et les règles de polarisation.

Le postulat énoncé a trouvé un premier champ d'application dans l'interprétation de la polarisation de la lumière de résonance optique $\left(^{4}\right)$. Il a permis d'appliquer à ce phénomène les schémas Zeeman et d'en déduire les règles de polarisation pour les transitions $\Delta M=0$ et \pm 1 . Ces conclusions sont bien conformes à l'expérience, que la lumière incidente soit naturelle, rectiligne ou circulaire.

Application à la diffusion de la lumière. - Rappelons l'essentiel des faits : on éclaire un milieu diffusant avec de la lumière rectiligne, circulaire ou naturelle et l'on observe la lumière diffusée soit à angle droit, soit parallèlement au faisceau incident.

a) lumière incidente rectiligne, observation à angle droit. On définit le facteur de dépolarisation des raies Rayleigh ou Raman $: p=\frac{i}{\mathbf{I}}$. On a toujours $0<p<\frac{3}{\mathbf{4}}$.

b) lumière incidente circulaire, observation parallèle au faisceau incident (Expériences de Hanle et Bär). On observe dans ce cas une inversion partielle de polarisation circulaire. On définit un rapport d'inversion $r=\frac{I_{v}}{I_{r}}$.

On a toujours $0<r<6$.

Entre $r$ et $p$ on a la relation $\frac{2}{r}=\frac{1}{p}-1$.

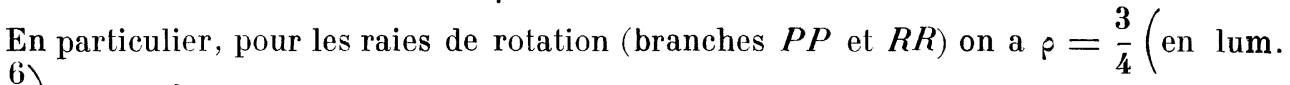
nat. $\frac{6}{7}$ ) et $r=6$.

Ces résultats déduits par Cabannes de la théorie classique ont été déjà en partie vérifiés par l'expérience (ち). Nous allons voir qu'ils découlent également du bilan de moment cinétique correctemerit appliqué. De tels essais d'interprétation ont été faits récemment (6) mais sont restés en désaccord avec les résultats de la théorie classique, (ainsi, leurs auteurs trouvent $r=\infty$ au lieu de $r=6$ pour les raies de rotation. Le désaccord provient de ce que, dans ces essais, le bilan de moment cinétique a été appliqué au vecteur $\vec{J}\left({ }^{*}\right)$.

$0^{\prime}$, , le pos/ulat de Heisenberg montre nettement que ce bilan doit être appliqué au vecteur $\vec{M}$ (composante de $\vec{J}$ suivant l'axe privilégié) et non pas au vecteur $\vec{J}$. Nous allons montrer, en rectifiant ainsi le bilan du moment cinétique, que les règles de polarisation conduisent au même résultat que les calculs de Cabannes et Rocard.

Nous pouvons considérer schématiquement avec Smekal le processus de diffusion de la lumière comme une succession de deux sauts simples $(p \rightarrow r \rightarrow q$, absorption du quantum incident suivi de réémission); à chaque saut parliel $p \rightarrow r, r \rightarrow q$, s'applique la règle de sélection $\Delta J= \pm 1$. (La transition $\Delta J=0$ est interdite, au moins pour les molécules linéaires). Il en résulte pour la diffusion la règle de sélection $\Delta J=0$ ou \pm 2 ; les transitions $\Delta J=0$ fournissent la branche $Q$; les transitions $\Delta J= \pm 2$ correspondent respectivement aux branches $R R$ et $P P\left({ }^{* *}\right)$.

(*) Ces notations se rapportent au cas ( $a$ ) de Hund. Pour les molécules se trouvant dans le cas (b), il suffirait de remplacer $J$ par $K$ et $M$ par $M_{l}$. Toutes les conclusions sont les mêmes.

$\left.{ }^{\star \star}\right)$ Dans les molécules non linéaires les transitions $p \rightarrow r$ ou $r \rightarrow q$ peuvent correspondre aussi à $\Delta J=0$, ce qui fournit aussi des branches Raman $\Delta J= \pm 1$ qui ont été trouvées dans l'effet Raman de $\mathrm{NH}^{2}$. Les notations en usage sont $Q(\Delta j=0), R R$ ou $S(\Delta j=+2), P P$ ou $O(\Delta j=-2), R(\Delta j=+1)$ et $P(\Delta j=-1)$. 
Pour aborder les règles de polarisation il faut tracer pour chaque niveau $p, r, q$, le schéma des sous-niveaux $M$. Chaque niveau caractérisé par le nombre quantique $J$ se subdivise en $2 J+1$ sous-niveaux magnétiques caractérisés par les nombres $\boldsymbol{M}=-J$, $-J+1, \ldots, J-1, J$. La figure 2 montre le schéma des nombres $M$ pour un niveau $J=3$, et les 7 orientations correspondantes du vecteur $J$ dans l'espace, $x x^{\prime}$ étant l'axe de révolution. Le vecteur $J$ est toujours positif par définition, mais sa projection $\boldsymbol{M}$ peut ètre soit positive, soit négative. Pour bien saisir le caractère vectoriel du bilan du moment cinétique, il faut caractériser le moment cinétique de la lumière incidente également par un vecteur que je désigne par $\vec{\mu}= \pm 1$ ou 0 (en unités $\frac{h}{2 \pi}$ ) et dirigé suivant la droite $x^{\prime} x$.

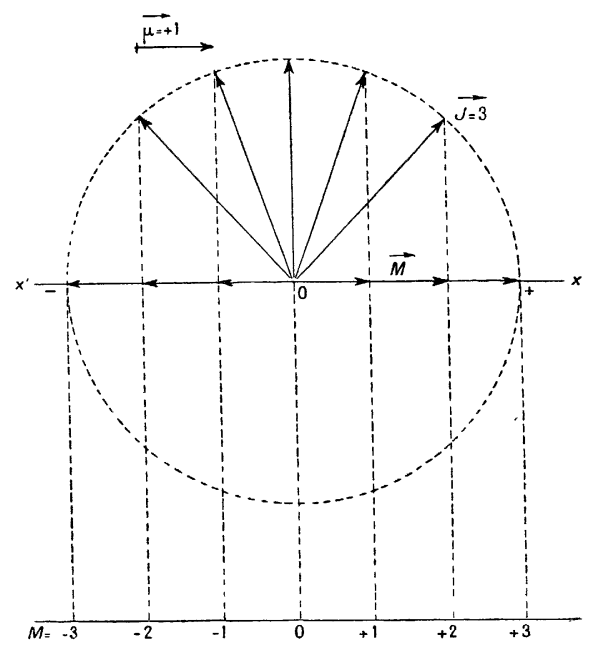

Fig. 2.

$\vec{\mu}=+1$ correspond à la lumière circulaire droite

$\vec{\mu}=-1$ correspond à la lumière circulaire gauche

$\vec{\mu}=0$ correspond à la lumière rectiligne.

'Nous caractérisons de même la lumière diffusée par le vecteur $\vec{\mu}$ '. Le bilan de moment cinétique se traduit alors par les deux identités vectorielles : $\vec{\mu}=\overrightarrow{\Delta M_{1}}($ transition $p \rightarrow r)$ et $\overrightarrow{\mu^{\prime}}=-\overrightarrow{\Delta M}_{2}$ (transition $r \rightarrow q$ ). L'état de polarisation de la lumière incidente fixe la variation $\overrightarrow{\Delta M}_{1}$ de la première transition tandis que le signe de la variation $\overrightarrow{\Delta .}_{2}$ de la deuxième transition détermine à son tour l'état de polarisation de la lumière diffusée. Le schéma de la figure 3 correspond à une raie de la branche $R \boldsymbol{R}(J \rightarrow J+2)$. Il permet de se représenter les divers cas possibles. Soit $0 \mathrm{X}$ - la direction de la lumière incidente.

Le $\operatorname{cas} \alpha$ correspond à de la lumière incidente rectiligne (supposée vibrant suivant $0 z$ ); son absorption (flèche $A_{1}$ ) entraîne la diffusion de 3 parts de lumière : $A_{2}$, lumière rectiligne parallèle à $A_{1}$ et qui contribue à la partie polarisée $I ; B_{2}$ et $C_{2}$ lumières circulaires inverses, dont le mélange $\frac{B_{2}+C_{2}}{2}$ observé suivant $o y$, contribue à la partie dépolarisée $i$.

Le cas $\beta$ schématise la diffusion obtenue en éclairant par la lumière circulaire droite 
$B_{1}$. On voit que, dans ce cas, la lumière diffusée se compose encore de 3 parts : $B_{2}$ donne une vibration circulaire régulière, $C_{2}$ une vibration circulaire inversée, mais en plus on obtient une vibration rectiligne $A_{2}$ qui, observée à angle droit de la direction incidente, fournit la part dépolarisée $i$.

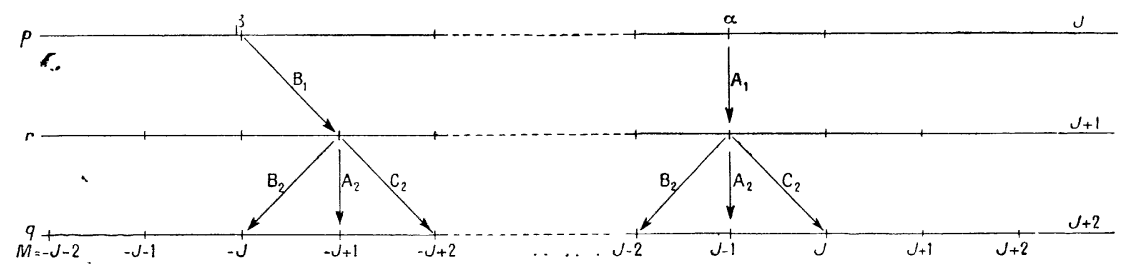

Fig. 3 .

Pour obtenir les diverses composantes $\left(I, i I_{r}, I_{v}\right)$ d'une raie de diffusion, il faut considérer chaque point $M$ du niveau initial $p$ comme point de départ d'un système de flèches et additionner les intensités do tous les éléments homologues. Comme toutes les orientations initiales sont également probables, l'intensité de chaque part est proportionnelle au produit $\boldsymbol{P}_{1} \times \boldsymbol{P}_{2}$ des deux probabilités de passage successives qui lui donnent naissance. Ces probabilités de passage ne sont autres que celles des composantes Zeeman des spectres de bandes. Les voici en unités arbitraires (en supprimant, pour simplifier, un facteur commun indépendant de $\boldsymbol{M})\left(^{\star}\right)$.

$$
\begin{aligned}
& J \rightarrow J+1 \begin{cases}M \rightarrow M+1 & P=(J+M+1)(J+M+2) \\
M \rightarrow M & P=2(J+M+1)(J-M+1) \\
M \rightarrow M-1 & P=(J-M+1)(J-M+2)\end{cases} \\
& J \rightarrow J \begin{cases}M \rightarrow M+1 & P=(J+M+1)(J-M) \\
M \rightarrow M & P=2 M^{2} \\
M \rightarrow M-1 & P=(J-M+1)(J+M)\end{cases} \\
& J \rightarrow J-1 \begin{cases}M \rightarrow M+1 & P=(J-M)(J-M-1) \\
M \rightarrow M & P=2(J+M)(J-M) \\
M \rightarrow M+1 & P=(J+M)(J+M-1)\end{cases}
\end{aligned}
$$

Explicitons le calcul dans quelques cas simples :

$1^{\circ}$ Première raie de rotation de la branche $R R(J=0 \rightarrow J=2)$.

\section{a) Lumiére incidente rectiligne (fig. 4).}

La probabilité de passage $P_{a}$ étant la même pour les trois parts, les intensités des trois parts sont proportionnelles à :

$$
P_{b}=2.2 .2=8 \quad P_{c}=P_{d}=3.2=6
$$

$\left(^{\star}\right)$ Voir ces expressions dans De Kronig, Band Spectra and Molecular Strueture, p. 100 ou dans Ruark and Urey, Atoms, Molecules, Quanta, p. 708. Les facteurs communs omis, indépendants de $M$, se seraient éliminés de toute façon en faisant les rapports $\frac{i}{l}$ et $\frac{I v}{I r}$. Ces facteurs sont fonctions des sauts $\left(J, J^{\prime}\right)$ et $(\Lambda$, $\left.\Lambda^{\prime}\right)$. Il faut remarquer toutefois que l'un do ces factours peut devenir nul, et que la raie correspondante disparait alors. Ainsi la transition $\Delta j=0$ a une probabilité nulle pour le saut $1 \Sigma \rightarrow \mid \Sigma$. Il en résulte que les molécules dont l'état normal est $! \Sigma$, ne donnent pas de branches Raman $\Delta j= \pm 1$. 
d'où :

$$
\left.\begin{array}{l}
l \text { proportionnel à } P_{b}=8 \\
i \quad-\quad \frac{P_{c}+P_{d}}{2}=6
\end{array}\right\} \rho=\frac{3}{4}
$$

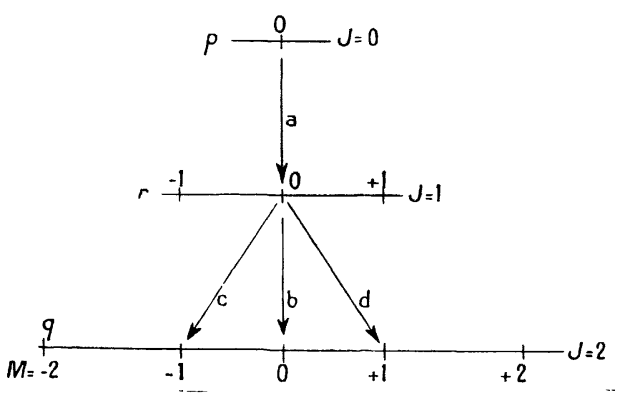

Fig. 4.

ß) Lumière incidente circulaire droite (fig. 5).

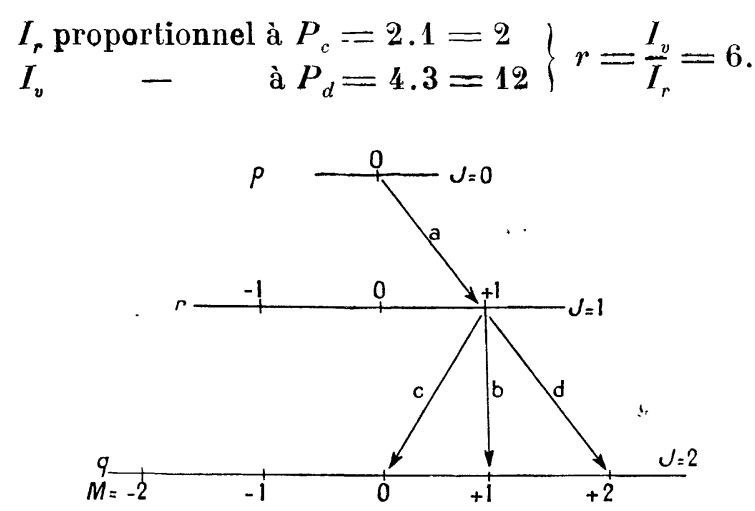

Fig. 5.

En observant à angle droit, on mesure :

$$
\left.\begin{array}{l}
I=\frac{I_{r}+I_{v}}{2}=7 \\
i=H_{b}=2.3 .1=6
\end{array}\right\} \rho=\frac{i}{I}=\frac{6}{7}
$$

$2^{\circ}$ Faisons encore le calcul de $r$ pour la deuxième raie de rotation $J=1 \rightarrow J=3$, dont la figure 6 montre le schéma :

En numérotant les transitions nous voyons que :

$$
\frac{I_{v}}{I_{r}}=\frac{P_{1} . P_{4}+P_{2} . P_{5}+P_{3} P_{6}}{P_{1} \cdot P_{7}+P_{2} . P_{8}+P_{3} . P_{9}}=\frac{1.2 .3 .4 .+2.3 .4 .5 .+3.4 .5 .6 .}{1.2 .4 .3 .+2.3 .3 .2 .+3.4 .2 .1 .}=6 .
$$

On généralise facilement ces formules $\left(^{\star}\right)$. On voit que les résultats s'accordent bien (*) Voir l'appenåice. 
avec ceux que Cabannes a déduits de la théorie classique. Tous ces résultats ont d'ailleurs déjà été déduits de la théorie générale de la diffusion développée à partir de la mécanique ondulatoire par Manneback et Placzek et Teller (7) mais j'ai voulu les établir ici sur des exemples très simples et d'une manière aussi élémentaire que possible, en les rattachant directement au bilan de moment cinétique et aux règles de polarisation appliquée au vecteur $\boldsymbol{M}\left(^{\star}\right)$.

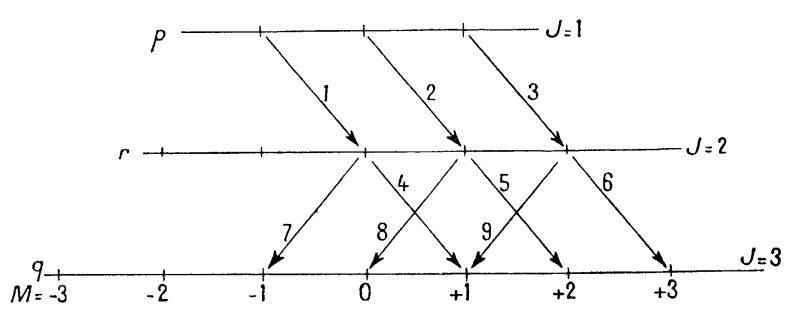

Fig. 6.

La figure 7 illustre un dernier exemple relatif à une raie $Q(\Delta J=0)$. On voit que si les états initial et final, sont tous les deux simples $(J=M=0)$ il ne peut y avoir ni dépolarisation, ni inversion de polarisation circulaire; la dépolarisation d'une raie Reyleigh ou Raman $Q$ est donc une conséquence directe de la subdivision, soit du niveau initial, soit du niveau final, en plusieurs sous-niveaux $M$ (dégénérescence).

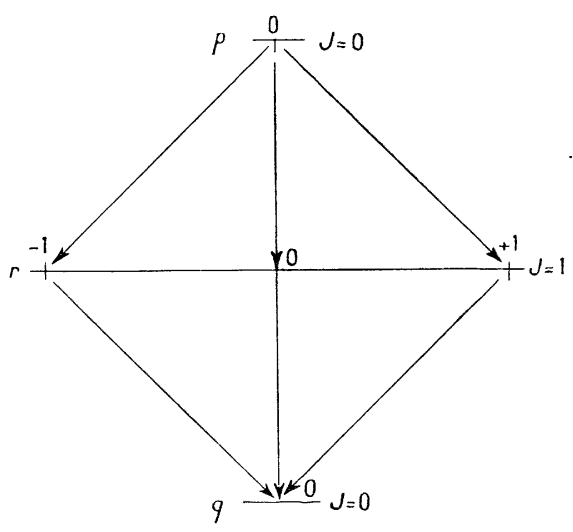

Fig. 7.

Elucidons un dernier point : Raman et Bhagavantam, en appliquant le bilan de moment cinétique au vecteur $\vec{J}$, avait montré que dans certaines de ces orientations initiales la molécule était incapable d'accepter le moment cinétique de la lumière incidente (४). Cela résulte immédiatement du caractère vectoriel de ce bilan.

${ }^{\star}{ }^{*}$ Appliqué aux raies Raman des branches $R$ et $P(\Delta j=: \pm 1$, résultant des sauts $p \rightarrow r \rightarrow q$ : $0 \pm 1$ ou $\pm 1+0)$, le mode de calcul ici exposé cenduit à des valeurs anormales de l'état de polarisation $\vdash \neq \frac{3}{4}$ et $r \neq 6$ ). En tenant compte d'une remarque de Placzek et Teller (loc. cit. p. 257, $\S b$ ) il faut sans doute conclure que ce mode de calcul n'est rigoureux qu'en cas de résonance (fréquence incidente $=$ fréquence d'absorption). 


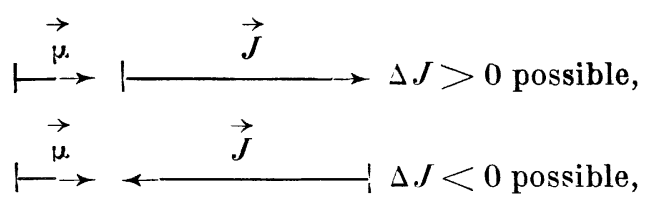

$$
\begin{aligned}
& \Delta . J<0 \text { impossible } \\
& \Delta J>0 \text { impossible. }
\end{aligned}
$$

En n'envisageant que des orientations parallèles ou antiparallèles des vecteurs $\vec{\mu}$. et $\vec{J}_{\text {, }}$ on voit en effet que la moitié des cas d'orientation serait « inefficace ».

Mais tout change si, au lieu d'appliquer ce bilan au vecteur $\vec{J}$, nous l'appliquons à sa composante $\vec{M}$. Le vecteur $\vec{J}$ peut présenter alors $2 J+1$ orientations différentes par rapport au rayon lumineux et $J$ et $|\boldsymbol{M}|$ peuvent donner des variations de signes contraires. Deux cas sont alors à distinguer :

a) Branche $R R: \Delta . J>0$. Toutes les orientations initiales peuvent servir de point de départ vers les $J$ supérieurs. Mais il est intéressant de remarquer (fig. 4, 5 et 6 ) que toutes les orientations du niveau final ne sont pas réalisées.

b) Branche $P P: \Delta J<0$. Comme dans chaque niveau $|M|<J$ on voit que deux transitions sont impossibles si la lumière incidente est polarisée circulairement ou rectilignement.

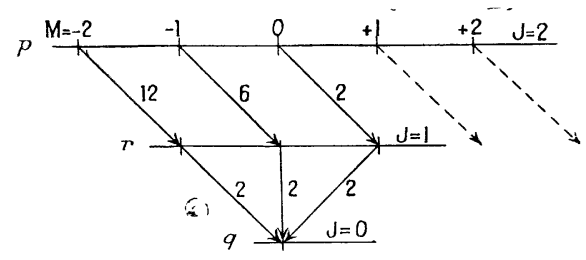

Fig. 8. - Les nombres inscrits à côté des flèches représentent les probabilités des transitions correspondantes.

La figure 8 montre un exemple où les orientations $\boldsymbol{M}=+1$ et $+2 \mathrm{du}$ niveau initial $(J=2)$ sont inefficaces. On peut remarquer que la proportion d'orientations inefficaces est faible pour les valeurs élevées de $J$. Il n'est donc pas possible d'adopter le point de vue de Raman et Bhagavantam, et selon lequel la moitié des transitions serait interdite par l'orientation relative des vecteurs $\vec{\mu}$ et $\vec{J}$.

On voit que l'inefficacité de certaines orientations du niveau initial (ici $J=2$ ) est une simple conséquence des règles de polarisation. C'est précisément en tenant compte de ce fait qu'on trouve les valeurs exactes :

$$
\begin{gathered}
\rho=\frac{6.2}{\frac{1}{2}[12.2+2.2]}=\frac{6}{7} \\
r=\frac{12.2}{2.2}=6 .
\end{gathered}
$$

et

Dans ces conditions, il devient difficile de suivre les considérations que les autenrs hindous ont échafaudées sur ce point, et par lesquelles ils cherchent à mettre en opposition le concept du (Spinning photon» avec les résultats de la théorie classique. La théorie quantique et la théorie classique se complètent et ne s'opposent pas ( $\left.{ }^{\star}\right)$.

Il reste cependant un point obscur : c'est le vecteur $\vec{J}$ qui mesure le moment cinétique

${ }^{\star}{ }^{\star}$ Voir aussi la remarque de Placzek et Teller. [(7), note de la page 2/48].

28. 
total de l'atome ou de la molécule. Le vecteur $\vec{M}$ indique seulement la valeur de sa projection sur l'axe $x^{\prime} x$ fixe dans l'espace. Pourquoi le principe de conservation du moment cinétique s'applique-t-il seulement à $\vec{M}$ et non pas à $\vec{J}$ ? La difficulté provient sans doute de ce que nous appliquons à un acte microscopique (action d'un seul photon sur un atome individuel) un concept comme celui du " moment cinétique » qui est peut être valable dans le domaine macroscopique seulement (action d'une onde lumineuse sur un grand nombre d'atomes).

Bilan de moment cinétique et fluorescence. - Le bilan de moment einétique et les règles de polarisation trouvent un champ d'application intéressant dans les phénomènes de fluorescences dûs à l'excitation optique des atomes par échelons successifs (9). Fuchtbauer et Wood ont montré qu'en éclairant de la vapeur de mercure simultanément à l'aide de deux sources indépendantes donnant l'une la raie de résonance $(\lambda=2537 \AA)$ et l'autre d'autres raies du mercure, on peut observer un spectre de fiuorescence contenant, à côté de la raie de résonance, de nombreuses raies du spectre de mercure. Ainsi, l'éclairement simultané par $\lambda=2537$ et la raie indigo $\lambda=4358 \AA$ produit à còté de la réémission de ces raies, l'émission des raies violette 4047 et verte 5461 du mercure. En voici l'explication : par l'absorption de la raie de résonance, l'atome $\mathrm{Hg}$ passe du niveau normal 1: $S_{0}$ au niveau excité $2^{3} P_{1}$, à partir duquel il devient capable d'absorber la raie indigo 4358 qui le porte au niveau plus élevé $2^{3} S_{1}$, point de départ des trois raies d'émission 4047, 4358 et วั461. En émettant la raie verte 5461 , l'atome tombe à l'état métastable $2^{3} P_{2}$.

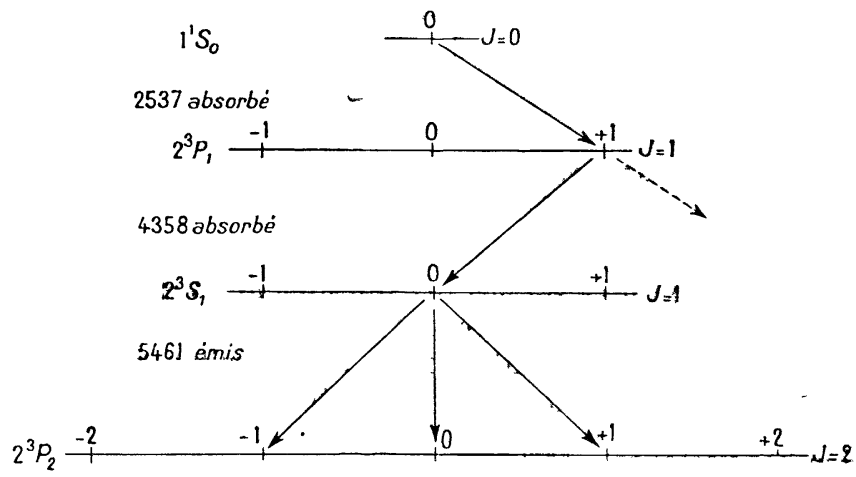

Fig. $9\left(^{*}\right)$.

La figure 9 montre le schéma dues nombres $\boldsymbol{M}$ de ces différontes transitions (pour chaque niveau $M=-J,-J+1, \ldots+. J)$.

Supposons maintenant que les lumières ineidentes 2537 et 4358 soient polarisées chacune circulairement. Si 2537 est circulaire droite seule la transition $\Delta M=+1$ sera possible qui conduit vers ${ }^{3} P_{1}(M=+1)$. On voit que si 4358 est également polarisé circulairement, son absorption n'est possible que si ses vibrations tournent dans l'espace en sens inverse de celle de $\left.2.377^{* *}\right)$. Si on étudie la fluorescence en éclairant successivement avec

${ }^{*}$ ) Il aurait été logique de dessiner les niveaux à des hauteurs proportionnelles â leur énorgie. Bans ce cas le sens de polarisation circnlaire aurait été indiqué parila pente de la flèche (- lextrorsum, + sinaistrorsum, dans l'émission et dans l'abserption). Mais pour no pas enchevetrer les figures, les niveaux sant lessinés de haut en bas dans leur ordre de succession. Le sens de la pente est donc renversé pour les raies d'absorplion.

$\left({ }^{* *}\right)$ Pendant les deux phases successives de l'absorption, les atomes conservent leur orientation invariable dans l'espace. Les différents sous-niveaux magnétique de $2^{3} P_{1}$ ne sont donc pas équivalents. C'est là une hypothèse essentielle. 
des lumières syu-et anticirculaires, on doit donc observer l'émission des raies 4047, 4.358 et 5461 dans le deuxième cas seulement.

En réalité, cette conclusion n'est qu'approchée car on sait que la raie de résonance n'est pas complètement polarisée ( $\left(^{*}\right)$. La figure 10 montre le schéma de l'expérience, il suffit d'utiliser le montage de Wood (10) en opérant avec des faisceaux de lumière circulaire (comme les deux faisceaux de lumière proviennent de deux directions opposées, ils doivent être polarisés circulairemént de même sens pour être anticirculaires l'un par rapport à l'autre).

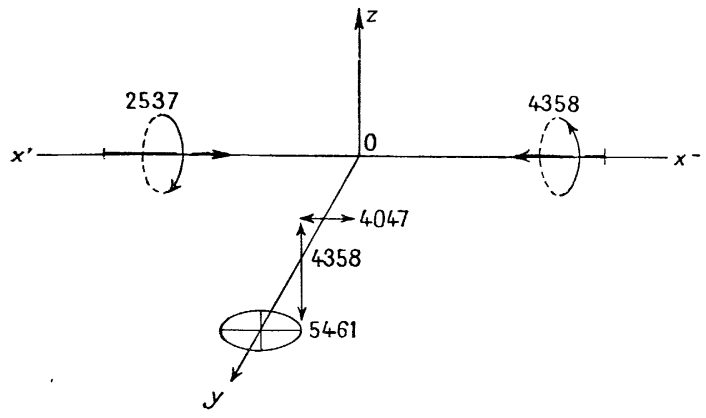

Fig. 10 .

L'émission de la raie verte $\mathbf{5} 461$ pourra se iaire par 3 transitions (de $M=0$ vers $M=$ $-1,0,+1)$. Cette raie sera donc à peu près dépolarisée.

Un cas plus intéressant est celui de la raie violette 4047 qui aboutit au niveau simple $2^{3} \mathrm{P}_{0}(J=M=0)$ et qui n'apparaitra par conséquent que dans la transition $M=0 \rightarrow M$ $=0$. Cétte raie de fluor escence devra donc être polarisée rectilignement, le vecteur électrique vibrant suivant $O X$ (c'est un cas de polarisation négative, $\rho=\infty$ ). On peut d'ailleurs, dans ce cas, imaginer plusieurs variantes de l'expérience qui fourniront autant de confirmations du postulat de Heisenberg. Leur schéma est donné dans la figure 11.
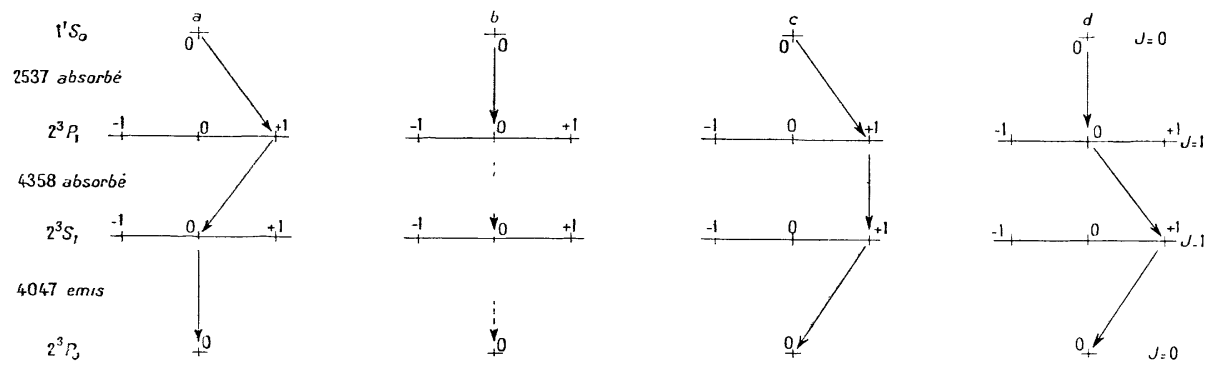

Fig. 11.

Le cas $a$ vient d'ètre étudié; dans le cas $b$ les deux faisceaux incidents sont polarisés rectilignement, vibrant suivant $o x$; la raie de fluorescence 4047, rectiligne devrait vibrer suivant $o x$ également. Mais nous allons voir que dans ce cas tout spectre de fluorescence doit disparaître.

Cas $c$ et $d$. L'une des lumières incidentes vibre rectilignement suivant $o x$ (la direction du faisceau étant $o z$ ) l'autre se propageant suivant $o x$ est circulaire. La lumière de fluorescence $(\Delta M=-1)$ observée suivant oy, paraîtra rectiligne vibrant suivant $o z$; observée suivant $x x^{\prime}$, elle sera circulaire et de même sens que la lumière incidente circulaire (obser-

(*) Une théorie complète du phénomène devra tenir compte de la structure hyperfine des raies. 
rer que la transition $\Delta M=+1$ correspond à des sens de circulation inverses dans l'absorplion et l'émission).

Ces indications sont à compléter par l'introduction des probabilités de passage. Dans les schémas ci-après (fig. 12) des radiations $2537,4358,4047$ et 5461 du mercure les nombres inscrits à côté des flèches représentent en unités arbitraires les probabilités de passage correspondantes calculées à partir du tableau donné plus haut.
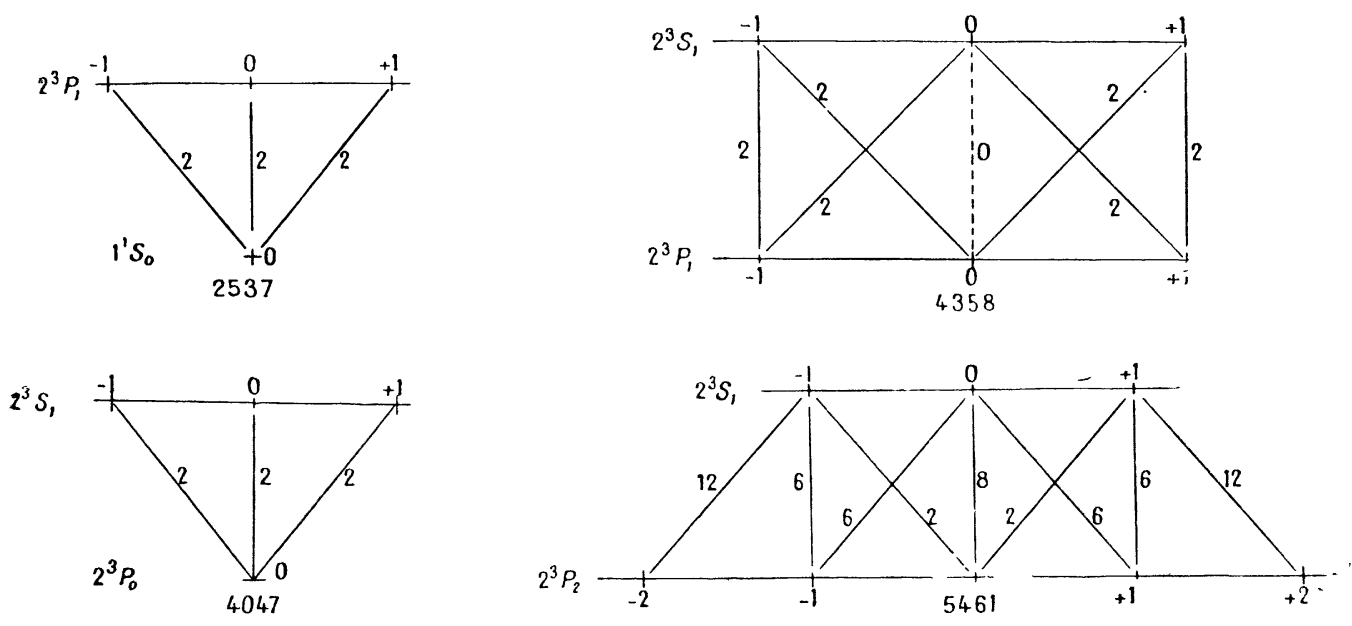

Fig. 12.

Dans le cas $a$ (fig. 9), en observant suivant oy, le degré de polarisation de la raie de Morescence 5461 doit donc être :

$$
\frac{I_{x}}{I_{z}}=\frac{8}{\frac{1}{2}(6+6)}=\frac{4}{3} .
$$

Pour les raies $\Delta J=0$ (c'est le cas de 4358 ) la transition $M=0 \rightarrow, I=0$ est interdite (sa probabilité de passage est nulle). Dans le cas b l'absorption de 4358 est donc impossible et tout spectre de fluorescence doit disparaitre.

Réémission de la raie 4358 . - Le schéma des probabilités de passage nous montre que dans les cas d'éclairement $c$ et $d$, cette raie de fluorescence donne $\rho=1$ et $r=0$.

Enfin la figure ci-jointe (fig.13) montre que dans le cas $a$, la réémission de $\mathbf{4 3} 8$ se fait par 2 vibrations circulaires inverses qui, vues suivant $o y$, apparaissent comme vibration rectiligne selon $o z$.

Lorsqu'on éclaire la vapeur de mercure avec les radiations anticirculaires 2.537 et 4358 . le spectre de fluorescence, observé à angle droit des faisceaux incidents contient les raies 4358 et 4047 polarisées rectilignement à angle droit, la première vibrant dans le plan d'onde des lunières incidentes, la deuxième suivant la normale à ce plan (voir fig. 10).

Ce résultat subsiste probablement lorsque les lumières incidentes sont naturelles pourvu ąu'elles aient même direction d'incidence et plar conséquent même plan d'onde $P\left({ }^{\star}\right)$.

Nous pouvons en effet considérer une vibration de lumière naturelle comme la superposition :

$1^{\circ}$ de 2 vibrations rectilignes perpendiculaires incohérentes

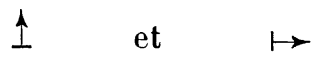

(*) Note ajoutée sur épreuves : Cette prévision a été vérifiée expérimentalement. 
$2^{\circ}$ de 2 vibrations circulaires inverses incohérentes

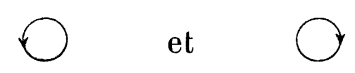

Cherchons alors tous les cas d'association possible de 2537 et 4358 :

a) En couplant $1 \rightarrow$ d'une part et (1) d'autre part il y a ambiguité dans l'appli- .

cation du postulat de Heisenberg, les 2 vibrations agissant successivement sur la molécule n'ayant pas même axe de révolution. Il est naturel d'admettre que ce couplage est contrarié et que l'intensité de la fluorescence correspondante est nulle.

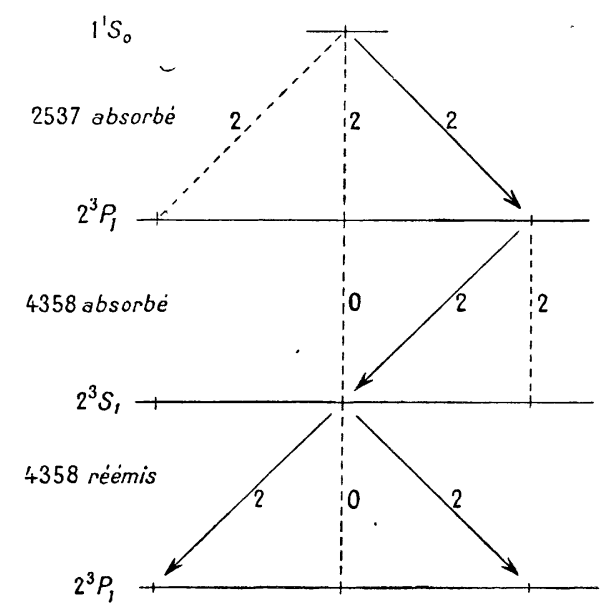

Fig. 13.

b) Restent les cas suivants de couplages conformes aux conditions de symétrie du phénomène :

$\downarrow \downarrow$ Nous retrouvons le cas $b$ inefficace.

$\bigcirc$ Lumières circulaires de mème sens, cas inefficace.

$\bigcirc 2$ paires de lumières anticirculaires. Cas a qui donne les seules couplages efficaces.

Rendement de fluorescence. - Remarquons enfin, que pour chaque raie de fluorescence, le rendement lumineux total doit être le même dans les 3 cas d'éclairement $a, c$ et $d$. Ceci résulte des valeurs des probabilités de passage et en particulier de la règle de somme de Burger et Dorgelo.

Le fait que la transition $M=0 \rightarrow M=0$ est interdite, permet de prévoir un cas curieux de fluorescence simple:

Supposons qu'un atome, dont l'état normal est un état ${ }^{1} S_{0}(J=M=0)$ soit excité en l'éclairant avec la lumière rectiligne d'une raie d'absorption $\Delta J=1\left({ }^{1} S_{0} \rightarrow{ }^{3} P_{1}\right)$, et qu'on provoque ainsi la réémission d'une raie de fluorescence $\Delta J=0\left({ }^{3} P_{1} \rightarrow{ }^{3} S_{1}\right)$.

Le schéma ci-après (fig. 14) montre qu'on devra mesurer un facteur de dépolarisation $\rho=\infty$. 


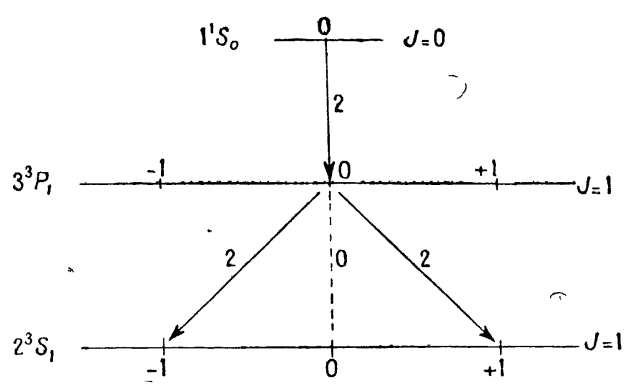

Fig. 14.

On peut trouver des exemples de ce genre dans les spectres d'arc des éléments bivalents. Ainsi pour le mercure l'absorption de la raie de résonance d'ordre supérieur $1436 \AA$ $\left(1^{1} S_{0} \rightarrow 3^{3} P_{1}\right)$ doit entraîner l'émission de la raie de fluorescence $13673 \AA\left(3^{3} P_{1} \rightarrow 2^{3} S_{1}\right)$.

Un autre exemple de ce genre se trouve réalisé dans la fluorescence visible de la vapeur de mercure lorsque celle-ci est mèlée d'azote. C'est alors l'état métastable $2^{3} P_{0}$, formé par choc à partir de $\mathbf{2}^{3} P$, qui joue le rôle de niveau initial. Les atomes métastables, en absorbant $4046 i(\Delta J=1)$ sont portés au niveau $2^{3} S$. La polarisation négative $(p>1)$ de la raie $4358(\Delta J=0)$, émise à partir de ce niveau, a été vérifiée (11).

\section{BIBLIOGRAPHIE}

(1) Rubivowicz, Physik, Z., 19 (1918), 441 et 465.

(2) Voir Sommerfedd, Atombau u. Spektrallinien, 5* chap. ₹ 2, Auswahlprinzip u. Polarisations-regel.

(3) Heisenberg, $Z$. Physik, 31 (1925), 617.

(4) Voir Privgshem, Fluorescenz u. Phosphorescenz, p. 98, 6 de la collection “Struktur der Materie ».

(5) Cabannes et Rocard, Journal de physique, 10 (1929), 52; J. Cabannes, Journal de Physique. 2 (1931), 381 ; S. Bhagavantam, Indian J. Phys., 6 (1931), 389.

(6) Raman et Bhagavantam, Nature, 128, 19 juillet et 26 septembre 1931, et Indian J. Phys., 6 (1931), 353 ; A. Kastler, $C$. R., 193 (1931), 1075.

( ${ }^{7}$ ) C. Manneback, Z. Physik, 62 (1930), 224 ; Plagzeck et Teller, Z. Physik, 81 (1933), 209.

$\left(^{8}\right)$ R.mar et Bhagavartam, Ind, Phys., 6 (1931), p. 358.

(9) Pringsheim, loc. cit., p. 26, Stufenweise Anregung; G. Bruhat, Cour d'Optique, p. 630-631.

(10) Pringsueim, loc. cit., p. 27; Brulita, loc. cit., p. 631 .

(11) Richter. Ann. der Physik, 7 (1930), 293; Mitcheld, Physical Review, 40 (1932), 964 et 43 (1933), 887.

\section{Appendice.}

Pour les raies de la branche $R R(\Delta J=+2)$, on établit facilement, à l'aide du tableau des probabilités de passage et en employant les notations de la figure 3 , les expressions générales suivantes :

$$
\begin{aligned}
\rho=\frac{i}{I}=\frac{\frac{1}{2}\left[\sum P\left(A_{1}\right) \times P\left(B_{2}\right)+\sum P\left(A_{1}\right) \times P\left(C_{2}\right)\right]}{\sum P\left(A_{1}\right) \times P\left(A_{2}\right)}=\frac{\sum P\left(A_{1}\right) \times P\left(C_{2}\right)}{P\left(A_{1}\right) \times P\left(A_{2}\right)} \\
=\frac{\sum_{M=-J}^{M=+J} 2(J+M+1)(J-M+1)(J+M+2)(J+M+3)[2 J+1 \text { termes }]}{M=+J} \\
\sum_{M=-J}^{M}(J+M+1)(J-M+1)(J+M+2)(J-M+2)[2 J+1 \text { termes }]
\end{aligned}
$$




$$
\begin{aligned}
& r=\frac{I_{v}}{I_{r}}=\frac{P\left(B_{1}\right) \times P\left(C_{2}\right)}{P\left(B_{1}\right) \times P\left(B_{2}\right)} \\
& =\frac{\sum_{y=-J}^{y=+J}(J+M+1)(J+M+2)(J+M+3)(J+M+4)[2 J+1 \text { termes }]}{\sum_{M=-J}(J+M+1)(J+M+2)(J-M+1)(J-M+2)[2 J+1 \text { termes }]} .
\end{aligned}
$$

Posons :

$$
\begin{aligned}
& U=\sum_{\boldsymbol{M}=-J}^{\boldsymbol{M}=\boldsymbol{J}}(J+\boldsymbol{M}+\mathbf{1})(J+\boldsymbol{M}+2)(J+\boldsymbol{M}+3)(J-\boldsymbol{M}+\mathbf{1}) \\
& V=\sum_{M=-J}^{M=+J}(J+\boldsymbol{M}+\mathbf{1})(J+\boldsymbol{M}+\mathbf{2})(J+\boldsymbol{J}+\mathbf{J})(\boldsymbol{J}+\boldsymbol{M}+\mathbf{4}) \\
& W=\sum_{M=-J}^{M=+J}(J+M+1)(J+M+2)(J-M+2)(J-M+1)
\end{aligned}
$$

Nous avons, avec ces notations :

$$
\rho=\frac{1}{2} \frac{U}{W} \quad \text { et } \quad r=\frac{V}{W} .
$$

Faisons. le changement de variable :

d'où :

$$
\begin{aligned}
& J+M=p \\
& J-M=2 J-p .
\end{aligned}
$$

A la variation de $M$ de $-J$ à $+J$ correspond la variation de l'entier $p$ de 0 à $2 J$. Donc :

$$
\begin{aligned}
& U=\sum_{0}^{2 J}(p+1)(p+2)(p+3)(2 J+1-p) \\
& V=\sum_{0}^{2 J}(p+1)(p+2)(p+3)(p+4) \\
& W=\sum_{0}^{2 J}(p+1)(p+2)(2 J+2-p)(2 J+1-p) .
\end{aligned}
$$

La théorie des combinaisons nous permet d'évaluer ces expressions. Rappelons les formules :

$$
\begin{gathered}
C_{p+k}^{k}=\frac{(p+k)(p+k-1)(p+k-2) \ldots(p+1)}{k !} \\
C_{k}^{k}+C_{k+1}^{k}+C_{k+2}^{k}+\ldots C_{k+l}^{k}=C_{k+l+1}^{k+1} .
\end{gathered}
$$

$1^{\circ}$ Calcul de $V$. - Le terme général de $V$ est :

$$
\begin{gathered}
(p+4)(p+3)(p+2)(p+1)=4 ! C_{p+4}^{4} \\
V=\sum_{0}^{2 J} 4 ! C_{p+4}^{4}=4 !\left(C_{4}^{4}+C_{5}^{4}+C_{6}^{4}+\ldots C_{2 J+4}^{4}\right)=4 ! C_{2 J+5}^{5} \\
V=\frac{1}{5}(2 J+5)(2 J+4)(2 J+3)(2 J+2)(2 J+1) . \\
\hline
\end{gathered}
$$


2 Calcul de $U$. - Le terme général de $U$ est :

$$
\begin{aligned}
& (p+3)(p+2)(p+1)(2 . J+1-p) \\
& =(p+3)(p+2)(p+1)(2 J+1)-(p+3)(p+2)(p+1) p \\
& =(2 J+1) .3 ! C_{p+3}^{3}-4 ! C_{p+3}^{4} \\
& U=(2 J+1) \cdot 3 ! \sum_{0}^{2 J} C_{p+3}^{3}-4 ! \sum_{0}^{2 J} C_{p+3}^{4} \\
& =(2 J+1) \cdot 3 !\left[C_{3}^{3 .}+C_{4}^{3}+\ldots C_{2 J+3}^{3}\right]-4 !\left[C_{4}^{4}+C_{5}^{4}+\ldots C_{2 J+3}^{4}\right] \\
& =(2 J+1) \cdot 3 ! C_{2 J+4}^{4}-4 ! C_{2 J+4}^{5} \\
& =\frac{2 J+1}{4}(2 J+4)(2 J+3)(2 . J+2)(2 J+1)-\frac{1}{5}(2 J+4)(2 J+3)(2 J+2)(2 J+1) 2 . J \\
& U=\frac{1}{20}(2 . J+5)(2 . J+4)(2 J+3)(2 . J+2)(2 . J+1) .
\end{aligned}
$$

$3^{\circ}$ Calcul de $W$. - Le terme général de $W$ est :

$$
(p+2)(p+1)(2 . J+2-p)(2 J+1-p)
$$

$=(2 . J+2)(2 J+1)(p+2)(p+1)-(4 J+3)(p+2)(p+1) p+(p+2)(p+1) \cdot p$.

Or $(p+2)(p+1) p^{2}$ peut s'écrire $(p+2)(p+1)[p(p-1)+p]$, d'où le terme général :

$$
\begin{aligned}
& (2 J+2)(2 J+1)(p+2)(p+1)-(4 . J+2)(p+2)(p+1) p+(p+2)(p+1) p \cdot(p-1) \\
& =(2 . J+2)(2 . J+1) .2 ! C_{p+2}^{2}-2(2 . J+1) .3 ! C_{p+2}^{3}+4 ! C_{p+2}^{4} \\
& \boldsymbol{W}^{\prime}=(2 J+2)(2 J+1) \cdot 2 ! \sum_{0}^{2 J} C_{p+2}^{2}-2(2 J+1) \cdot 3 ! \sum_{0}^{2 J} C_{p+2}^{3}+4 \sum_{0}^{2 J} C_{p+2}^{4} \\
& =(2 J+2)(2 J+1) \cdot 2 ! C_{2 J+3}^{3}-2(2 J+1) \cdot 3 ! C_{2 J+3}^{4}+4 ! C_{2 J+3}^{5} \\
& =\frac{(2 J+2)(2 J+1)}{3}(2 J+3)(2 J+2)(2 J+1)-\frac{2 J+1}{2}(2 J+3)(2 J+2)(2 J+1) 2 J \\
& +\frac{1}{\grave{g}}(2 J+3)(2 J+2)(2 J+1)(2 . J)(2 J-1) \\
& =(2 J+3)(2 J+2)(2 . J+1)\left[\frac{(2 J+2)(2 J+1)}{3}-(2 J+1) J+\frac{1}{5} 2 . J(2 . J-1)\right] \\
& W^{\prime}=\frac{1}{30}(2 J+5)(2 J+4)(2 J+3)(2 J+2)(2 J+1) .
\end{aligned}
$$

On a bien :

$$
\rho=\frac{1}{2} \frac{U}{W}=\frac{1}{2} \cdot \frac{30}{20}=\frac{3}{4} \quad \text { et } \quad r=\frac{V}{W}=\frac{30}{\grave{5}}=6 .
$$

Nơus nous dispensons d'écrire les expressions correspondantes pour les raies de la branche $P P(\Delta J=-2)$ qui se calculeraient de la même façon. 